\title{
COMPUTATIONAL EFFECTIVENESS OF LMI DESIGN STRATEGIES FOR VIBRATION CONTROL OF LARGE STRUCTURES
}

\author{
Francisco Palacios-Quiñonero ${ }^{1}$, Josep Rubió-Massegú ${ }^{1}$, Josep M. Rossell ${ }^{1}$ and \\ Hamid Reza Karimi ${ }^{2}$
}

\author{
${ }^{1}$ CoDAlab, Department of Mathematics, Universitat Politècnica de Catalunya (UPC) \\ Av. Bases de Manresa 61-73, 08242 Manresa, Barcelona, Spain \\ e-mail: $\{$ francisco.palacios, josep.rubio, josep.maria.rossell $\} @$ upc.edu \\ ${ }^{2}$ Politecnico di Milano, Department of Mechanical Engineering, via La Masa 1, 20156 Milan, Italy \\ e-mail: hamidreza.karimi@polimi.it
}

Keywords: Computational Effectiveness, Structural Vibration Control, Seismic Protection, Linear Matrix Inequalities.

\begin{abstract}
Distributed control systems for vibration control of large structures involve a large number of actuation devices and sensors that work coordinately to produce the desired control actions. Design strategies based on linear matrix inequality (LMI) formulations allow obtaining controllers for these complex control problems, which are characterized by large dimensionality, high computational cost and severe information constraints. In this paper, we conduct a comparative study of the computational effectiveness of three different LMI-based controller design strategies: $H_{\infty}$, energy-to-peak and energy-to-componentwise-peak. The $H_{\infty}$ approach is a well-known design methodology and has been widely used in the literature. The energy-to-peak approach is a particular case of generalized $\mathrm{H}_{2}$ design that is gaining a growing relevance in structural vibration control. Finally, the energy-to-componentwise-peak approach is a less common case of generalized $\mathrm{H}_{2}$ design that produces promising results among the three considered approaches. These controller design strategies are applied to synthesize active state-feedback controllers for the seismic protection of a five-story building and a twentystory building both equipped with complete systems of interstory actuation devices. To evaluate the computational effectiveness of the proposed LMI design methodologies, the corresponding computation times are compared and a suitable set of numerical simulations is carried out to assess the performance of the obtained controllers. As positive results, two main facts can be highlighted: the computational effectiveness of the energy-to-peak control design strategy and the particularly well-balanced behavior exhibited by the energy-to-componentwise-peak controllers. On the negative side, it has to be mentioned the computational inefficiency of the considered LMI design methodologies to properly deal with very-large-scale control problems.
\end{abstract}




\section{INTRODUCTION}

Over the last years, controller design strategies based on linear matrix inequality (LMI) formulations have been attracting increasing interest [1]. In the field of vibration control of large structures, these design strategies allow obtaining optimal controllers for complex control problems that involve a large number of actuation devices and sensors, and are typically characterized by large dimensionality, high computational cost and severe information constraints [2-7].

An important family of controller design methodologies for linear systems is based on the idea of minimizing the worst-case gain from the disturbance-input $w(t)$ to the closed-loop controlled-output $z(t)$, which can be modeled as the value

$$
\sup _{\|w\|_{\mathrm{w}} \neq 0} \frac{\|z\|_{\mathrm{z}}}{\|w\|_{\mathrm{w}}}
$$

where $\|\cdot\|_{z}$ and $\|\cdot\|_{\mathrm{w}}$ denote particular signal norms defined on the controlled-output and disturbance-input sets, respectively. Broadly speaking, an optimal controller is computed by minimizing the worst-case gain and, simultaneously, demanding asymptotic stability of the closed-loop system. From a computational point of view, an important fact is that some of these optimization problems can be formulated in terms of LMIs and solved using the existing LMI solvers [8]. Following this approach, different control design strategies can be obtained by selecting different signal norms in the controlled-output and disturbance-input spaces. In the present work, three different signal norms are considered: (i) the continuous 2-norm

$$
\|f\|_{2}=\left[\int_{0}^{\infty} f^{T}(t) f(t) d t\right]^{1 / 2},
$$

which is commonly understood as the signal energy content, (ii) the usual continuous peaknorm

$$
\|f\|_{\infty}=\sup _{0 \leq t<+\infty}\left[f^{T}(t) f(t)\right]^{1 / 2}
$$

and (iii) the componentwise-peak-norm

$$
\|f\|_{\infty, \mathrm{cw}}=\sup _{0 \leq t<+\infty} \max _{i}\left|f_{i}(t)\right|=\max _{i} \sup _{0 \leq t<+\infty}\left|f_{i}(t)\right|,
$$

where $f(t)=\left[f_{1}(t), \ldots, f_{n}(t)\right]^{T}$ is a real vector of dimension $n$. The objective of the paper is to conduct a comparative study of the computational effectiveness of three different LMIbased controller design strategies: $H_{\infty}$, energy-to-peak and energy-to-componentwise-peak. The $H_{\infty}$ approach is a well-known design methodology that uses the 2-norm to measure both the controlled-output and the disturbance-input [9-15]. The energy-to-peak approach is a particular case of generalized $\mathrm{H}_{2}$ design [16] that is gaining a growing relevance in structural vibration control [17-20]. This second case uses the 2-norm for the disturbance-input and the peak-norm for the controlled-output. Finally, the energy-to-componentwise-peak approach is a less common case of generalized $H_{2}$ design, which uses the 2-norm for the disturbance input and the componentwise-peak-norm for the controlled-output [21]. These three controller design strategies are applied to synthesize active state-feedback controllers for the seismic protection of a five-story building and a twenty-story building equipped with complete systems of interstory actuation devices. To assess the performance of the obtained controllers, a proper set of numerical simulations is conducted and the computational effectiveness of the proposed LMI design methodologies is evaluated by comparing the corresponding computation times. 
The rest of the paper is organized as follows: In Section 2, a brief summary of the LMI formulations corresponding to the $H_{\infty}$, energy-to-peak and energy-to-componentwise-peak controller design strategies is presented. In Section 3, a mathematical model for a $n$-story building is provided. In Section 4, the controllers corresponding to the three proposed design strategies are computed, the computation times are compared and the results of the numerical simulations are discussed. Finally, some conclusions are presented in Section 5 .

\section{LMI CONTROLLER DESIGN STRATEGIES}

Let us consider the system

$$
\left\{\begin{array}{l}
\dot{x}(t)=A x(t)+B u(t)+E w(t) \\
z(t)=C x(t)+D u(t)
\end{array}\right.
$$

where $x(t) \in \mathbb{R}^{n_{x}}$ is the state, $u(t) \in \mathbb{R}^{n_{u}}$ is the control input, $w(t) \in \mathbb{R}^{n_{w}}$ is the disturbance input, $z(t) \in \mathbb{R}^{n_{z}}$ is the controlled output and $A, B, E, C, D$ are constant real matrices with appropriate dimensions. Given a state-feedback controller

$$
u(t)=G x(t),
$$

with state gain matrix $G \in \mathbb{R}^{n_{u} \times n_{x}}$, we obtain the closed-loop system

$$
\left\{\begin{array}{l}
\dot{x}(t)=A_{G} x(t)+E w(t) \\
z(t)=C_{G} x(t)
\end{array}\right.
$$

where

$$
A_{G}=A+B G, \quad C_{G}=C+D G .
$$

The worst-case gain from the disturbance-input to the closed-loop controlled-output associated to the control gain matrix $G$ can be modeled by the system norm

$$
\gamma(G)=\sup _{\|w\|_{\mathrm{w}} \neq 0} \frac{\|z\|_{\mathrm{z}}}{\|w\|_{\mathrm{w}}}
$$

where $\|\cdot\|_{\mathrm{z}}$ and $\|\cdot\|_{\mathrm{w}}$ denote particular signal norms defined on the controlled-output and disturbance-input spaces, respectively. An optimal state-feedback controller

$$
u(t)=\widetilde{G} x(t)
$$

can then be obtained by solving the following optimization problem:

$$
\min _{G}\left\{\gamma(G): A_{G} \text { asymptotically stable }\right\} .
$$

The $H_{\infty}$ controller design considers the system norm

$$
\gamma_{\infty}(G)=\sup _{\|w\|_{2} \neq 0} \frac{\|z\|_{2}}{\|w\|_{2}}
$$

where $\|\cdot\|_{2}$ is the continuous 2-norm in (2). The optimization problem (11) corresponding to this case can be formulated as the following LMI optimization problem [1]:

$$
\mathcal{P}_{\infty}:\left\{\begin{array}{l}
\text { maximize } \eta \\
\text { subject to } X>0, \eta>0 \text { and the LMI in (14) }
\end{array}\right.
$$




$$
\left[\begin{array}{cc}
A X+X A^{T}+B Y+Y^{T} B^{T}+\eta E E^{T} & (C X+D Y)^{T} \\
C X+D Y & -I_{n_{z}}
\end{array}\right]<0,
$$

where $X=X^{T} \in \mathbb{R}^{n_{x} \times n_{x}}$ and $Y \in \mathbb{R}^{n_{u} \times n_{x}}$ are the optimization variables and $I_{n}$ is the identity matrix of order $n$. If an optimal value $\widetilde{\eta}_{\infty}$ is attained in $\mathcal{P}_{\infty}$ for the pair $\left(\widetilde{X}_{\infty}, \widetilde{Y}_{\infty}\right)$, then the state gain matrix

$$
\widetilde{G}_{\infty}=\widetilde{Y}_{\infty} \widetilde{X}_{\infty}^{-1}
$$

is an optimal solution to the $H_{\infty}$ controller synthesis problem and the corresponding $\gamma$-value can be computed as

$$
\widetilde{\gamma}_{\infty}=\gamma_{\infty}\left(\widetilde{G}_{\infty}\right)=\left(\widetilde{\eta}_{\infty}\right)^{-1 / 2}
$$

The energy-to-peak controller design uses the system norm

$$
\gamma_{\mathbf{p}}(G)=\sup _{\|w\|_{2} \neq 0} \frac{\|z\|_{\infty}}{\|w\|_{2}}
$$

where $\|\cdot\|_{2}$ is the 2-norm in (2) and $\|\cdot\|_{\infty}$ is the peak-norm given in (3). The optimization problem (11) associated to this second case admits the following LMI formulation [16]:

$$
\begin{gathered}
\mathcal{P}_{\mathrm{p}}:\left\{\begin{array}{l}
\text { minimize } \eta \\
\text { subject to the LMIs in } 19) \text { and } 20
\end{array}\right. \\
A X+X A^{T}+B Y+Y^{T} B^{T}+E E^{T}<0, \\
{\left[\begin{array}{cc}
X & (C X+D Y)^{T} \\
C X+D Y & \eta I_{n_{z}}
\end{array}\right]>0,}
\end{gathered}
$$

with LMI variables $X=X^{T} \in \mathbb{R}^{n_{x} \times n_{x}}, Y \in \mathbb{R}^{n_{u} \times n_{x}}$. If an optimal value $\widetilde{\eta}_{\mathrm{p}}$ is attained in $\mathcal{P}_{\mathrm{p}}$ for the pair $\left(\widetilde{X}_{\mathrm{p}}, \widetilde{Y}_{\mathrm{p}}\right)$, then the state gain matrix

$$
\widetilde{G}_{\mathrm{p}}=\widetilde{Y}_{\mathrm{p}} \widetilde{X}_{\mathrm{p}}^{-1}
$$

is an optimal solution to the energy-to-peak controller synthesis problem with an associated $\gamma$-value

$$
\widetilde{\gamma}_{\mathrm{p}}=\gamma_{\mathrm{p}}\left(\widetilde{G}_{\mathrm{p}}\right)=\left(\widetilde{\eta}_{\mathrm{p}}\right)^{1 / 2}
$$

Finally, the energy-to-componentwise-peak controller design considers the system norm

$$
\gamma_{\mathrm{cwp}}(G)=\sup _{\|w\|_{2} \neq 0} \frac{\|z\|_{\infty, \mathrm{cw}}}{\|w\|_{2}}
$$

where $\|\cdot\|_{2}$ is the usual 2-norm and $\|\cdot\|_{\infty, \mathrm{cw}}$ is the componentwise-peak-norm defined in (4). The optimization problem corresponding to this third case

$$
\min _{G}\left\{\gamma_{\text {cwp }}(G): A_{G} \text { asymptotically stable }\right\}
$$

can be formulated as follows [16]:

$$
\mathcal{P}_{\text {cwp }}:\left\{\begin{array}{l}
\text { minimize } \eta \\
\text { subject to the LMIs in } 26 \text { and } 27
\end{array}\right.
$$




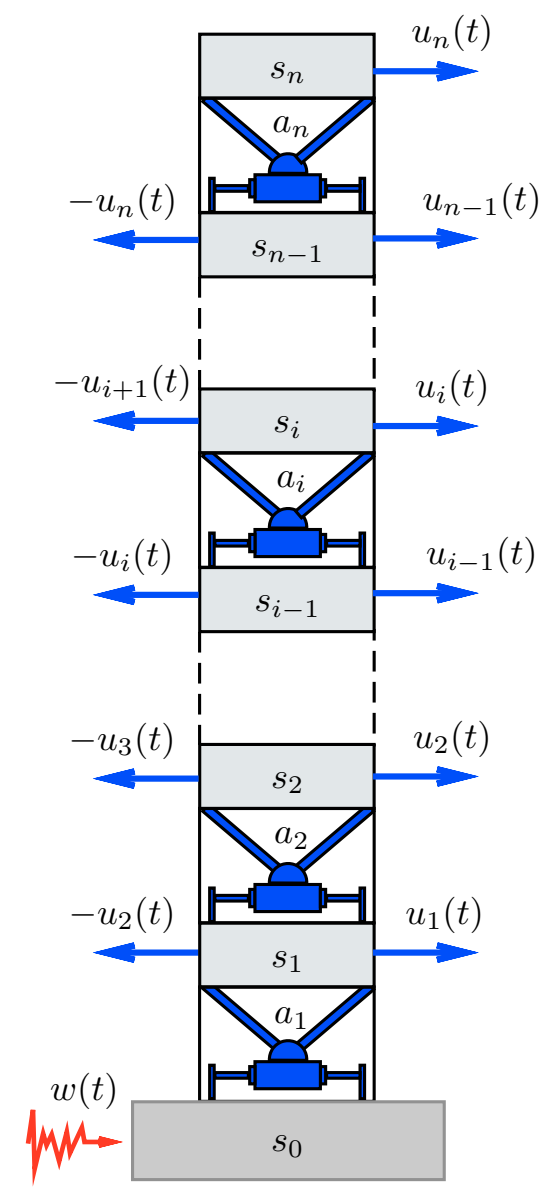

Figure 1: Building structure equipped with interstory actuation devices.

$$
\begin{gathered}
A X+X A^{T}+B Y+Y^{T} B^{T}+E E^{T}<0, \\
{\left[\begin{array}{cc}
X & \left(C_{i} X+D_{i} Y\right)^{T} \\
C_{i} X+D_{i} Y & \eta
\end{array}\right]>0, \quad \text { for } 1 \leq i \leq n_{z},}
\end{gathered}
$$

where $C_{i}$ and $D_{i}$ denote the $i$ th row of the matrices $C$ and $D$, respectively, and $X=X^{T} \in$ $\mathbb{R}^{n_{x} \times n_{x}}, Y \in \mathbb{R}^{n_{u} \times n_{x}}$ are the LMI variables. As it happened in the previous designs, if an optimal value $\widetilde{\eta}_{\text {cwp }}$ is attained in $\mathcal{P}_{\text {cwp }}$ for the pair $\left(\widetilde{X}_{\text {cwp }}, Y_{\text {cwp }}\right)$, then the state gain matrix

$$
\widetilde{G}_{\mathrm{cwp}}=\widetilde{Y}_{\mathrm{cwp}} \widetilde{X}_{\mathrm{cwp}}^{-1}
$$

is an optimal solution to the energy-to-componentwise-peak controller synthesis problem with an associated $\gamma$-value

$$
\widetilde{\gamma}_{\text {cwp }}=\left(\widetilde{\eta}_{\text {cwp }}\right)^{1 / 2}
$$

\section{BUILDING MODEL}

Let us consider the $n$-story building structure schematically depicted in Figure 1 , where $a_{i}$, $i=1, \ldots, n$, represents an interstory actuation device implemented between the stories $s_{i-1}$ and $s_{i}, w(t)$ denotes the seismic ground acceleration and $u_{i}(t)$ is the control action exerted by 
the actuator $a_{i}$, which produces a pair of opposite structural forces as indicated in the figure. By considering the vector of displacements

$$
q(t)=\left[q_{1}(t), \ldots, q_{n}(t)\right]^{T},
$$

where $q_{i}(t)$ is the lateral displacement of the story $s_{i}$ with respect to the ground level $s_{0}$, the lateral motion of the structure can be described by the differential equation

$$
M \ddot{q}(t)+C_{d} \dot{q}(t)+K q(t)=T_{u} u(t)+T_{w} w(t),
$$

where

$$
u(t)=\left[u_{1}(t), \ldots, u_{n}(t)\right]^{T}
$$

is the vector of control actions, $M, C_{d}$ and $K$ are the mass, damping and stiffness matrices, respectively, $T_{u}$ is the control location matrix and $T_{w}$ is the excitation input matrix. The mass matrix is a diagonal matrix

$$
M=\left[\begin{array}{llll}
m_{1} & & & \\
& \ldots & & \\
& & \ldots & \\
& & & m_{n}
\end{array}\right]
$$

and the stiffness matrix has the following tridiagonal structure:

$$
K=\left[\begin{array}{cccccc}
k_{1}+k_{2} & -k_{2} & & & & \\
-k_{2} & k_{2}+k_{3} & -k_{3} & & & \\
& \cdots & \ldots & \ldots & & \\
& & \cdots & \ldots & \ldots & \\
& & & -k_{n-1} & k_{n-1}+k_{n} & -k_{n} \\
& & & & -k_{n} & k_{n}
\end{array}\right]
$$

where $m_{i}$ and $k_{i}, i=1, \ldots, n$ denote the mass and stiffness coefficients of the $i$-th story, respectively. The damping matrix $C_{d}$ can be computed from $M$ and $K$ by setting a proper damping ratio on the building modes [22], the control location matrix is a square matrix of size $n$ with the following upper-diagonal band form:

$$
T_{u}=\left[\begin{array}{rrrrrr}
1 & -1 & & & & \\
& 1 & -1 & & & \\
& & \cdots & \ldots & & \\
& & & \ldots & \ldots & \\
& & & & 1 & -1 \\
& & & & & 1
\end{array}\right]
$$

and the excitation input matrix has the form

$$
T_{w}=-M[1]_{n \times 1},
$$

where $[1]_{n \times 1}$ denotes a vector of dimension $n$ with all its entries equal to 1 . By introducing the interstory drifts

$$
\left\{\begin{array}{l}
r_{1}(t)=q_{1}(t) \\
r_{i}(t)=q_{i}(t)-q_{i-1}(t) \quad \text { for } i=2, \ldots, n,
\end{array}\right.
$$




\begin{tabular}{lccccc}
\hline story & 1 & 2 & 3 & 4 & 5 \\
\hline mass $\left(\times 10^{5} \mathrm{Kg}\right)$ & 2.152 & 2.092 & 2.070 & 2.048 & 2.661 \\
stiffness $\left(\times 10^{8} \mathrm{~N} / \mathrm{m}\right)$ & 1.470 & 1.130 & 0.990 & 0.890 & 0.840 \\
relative damping & $5 \%$ & & & & \\
\hline
\end{tabular}

Table 1: Parameter values corresponding to the five-story building model.

and the augmented state vector

$$
x(t)=\left[\begin{array}{c}
r(t) \\
\dot{r}(t)
\end{array}\right]
$$

we obtain the following first-order state-space model:

$$
\dot{x}(t)=A x(t)+B u(t)+E w(t),
$$

with

$$
\begin{gathered}
A=P \widehat{A} P^{-1}, \quad B=P \widehat{B}, \quad E=P \widehat{E}, \\
\widehat{A}=\left[\begin{array}{cc}
{[0]_{n \times n}} & I_{n} \\
-M^{-1} K & -M^{-1} C_{d}
\end{array}\right], \quad \widehat{B}=\left[\begin{array}{c}
{[0]_{n \times n}} \\
M^{-1} T_{u}
\end{array}\right], \quad \widehat{E}=\left[\begin{array}{r}
{[0]_{n \times 1}} \\
-[1]_{n \times 1}
\end{array}\right],
\end{gathered}
$$

where $[0]_{n \times m}$ represents a zero-matrix of the indicated dimensions and $P$ is the change-of-basis matrix corresponding to the state transformation

$$
\left[\begin{array}{c}
r(t) \\
\dot{r}(t)
\end{array}\right]=P\left[\begin{array}{c}
q(t) \\
\dot{q}(t)
\end{array}\right] .
$$

Assuming that the control objective is to reduce the interstory drift values in the seismically excited building by means of moderate control actions, we also consider the controlled-output vector

$$
z(t)=C x(t)+D u(t)
$$

defined by the matrices

$$
C=\left[\begin{array}{cc}
I_{n} & {[0]_{n \times n}} \\
{[0]_{n \times n}} & {[0]_{n \times n}}
\end{array}\right], \quad D=\alpha\left[\begin{array}{c}
{[0]_{n \times n}} \\
I_{n}
\end{array}\right],
$$

where $\alpha$ is a scaling factor that compensates the different magnitude of interstory drifts and control forces.

\section{NUMERICAL RESULTS}

Let us consider the particular five-story building model [23] corresponding to the mass, stiffness and damping parameters presented in Table 1. For this small-building problem, we first solve the LMI optimization problem $\mathcal{P}_{\infty}$ in (13) with the matrices $A, B$ and $E$ in (40) and the controlled-output matrices in (44) with $n=5$ and the scaling factor $\alpha=10^{-7.3}$, obtaining the $H_{\infty}$ control gain matrix

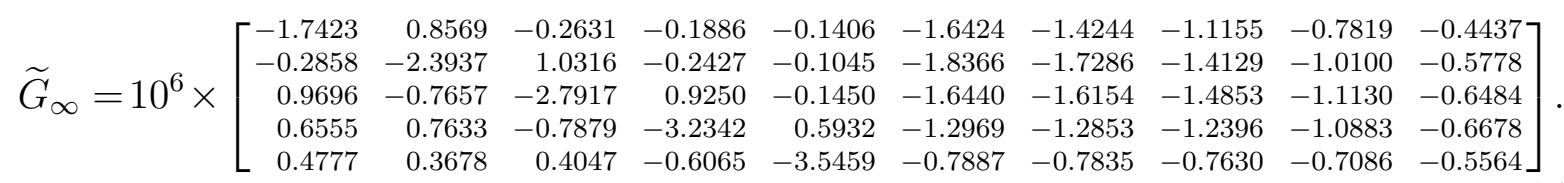




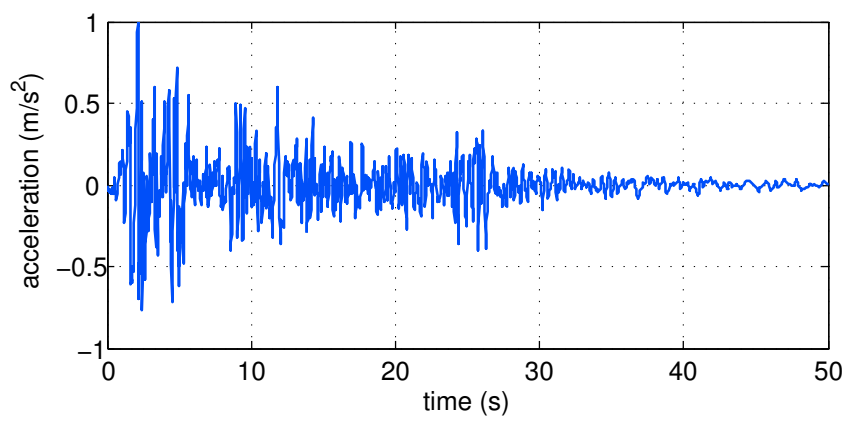

Figure 2: North-South El Centro 1940 seismic record scaled to $1 \mathrm{~m} / \mathrm{s}^{2}$.

\begin{tabular}{lccc}
\hline controller & $H_{\infty}$ & ETP & ETCWP \\
\hline computation time (s) & 0.6724 & 0.2105 & 0.3209 \\
\hline
\end{tabular}

Table 2: Computation time (in seconds) corresponding to the $H_{\infty}$, energy-to-peak (ETP) and energy-tocomponentwise-peak (ETCWP) control gain matrices obtained for the five-story building.

Next, we solve the optimization problem $\mathcal{P}_{\mathrm{p}}$ in $(18)$ with the same matrices $A, B, E$, and $C$ used in the previous design, and the matrix $D$ corresponding to the values $n=5$ and $\alpha=10^{-7.55}$. As a result, we obtain the following energy-to-peak control gain matrix:

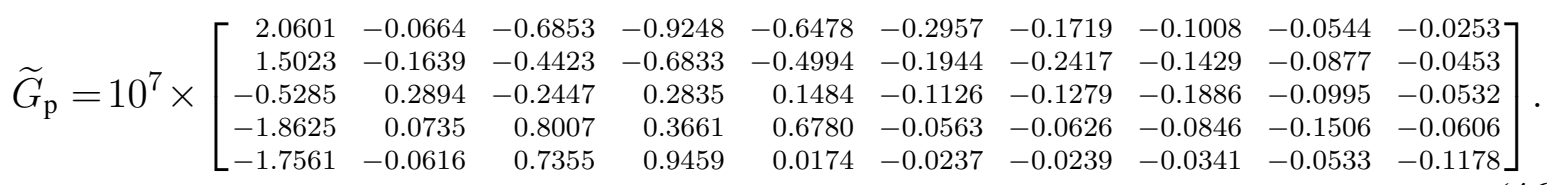

Finally, by solving the optimization problem $\mathcal{P}_{\text {cwp }}$ in 25 with the same matrices $A, B, E, C$ and $D$ used in the energy-to-peak design, we obtain the energy-to-componentwise-peak control gain matrix

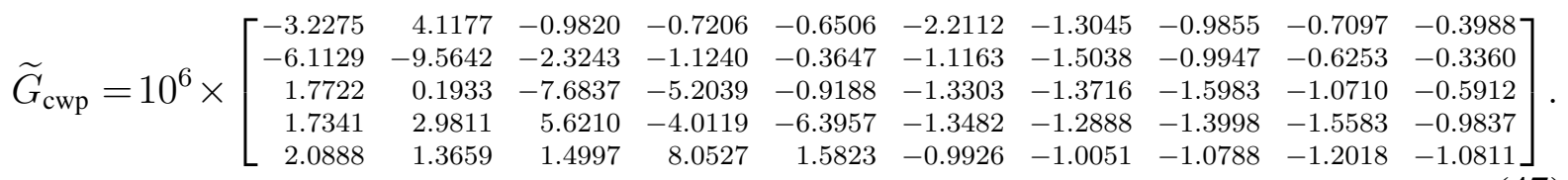

All the controllers in this section have been computed with the LMI solver included in the MATLAB Robust Control Toolbox [8] and using a personal computer with a two-core Intel i5 processor. The computation times corresponding to the control gain matrices $\widetilde{G}_{\infty}, \widetilde{G}_{\mathrm{p}}$ and $\widetilde{G}_{\text {cwp }}$ are collected in Table 2 .

To demonstrate the performance of the proposed controllers, a suitable set of numerical simulations has been carried out using the scaled North-South El Centro 1940 seismic record as a ground acceleration input (see Figure 2). The obtained absolute interstory-drift peak-values are displayed in Figure 3(a), where the black line with squares represents the uncontrolled response, the blue line with circles corresponds to the $H_{\infty}$ controller $u(t)=\widetilde{G}_{\infty} x(t)$, the green line with triangles presents the response of the energy-to-peak controller $u(t)=\widetilde{G}_{\mathrm{p}} x(t)$ and the red line with asterisks describes the energy-to-componentwise-peak controller $u(t)=\widetilde{G}_{\mathrm{cwp}} x(t)$. The corresponding absolute control-effort peak-values are displayed in Figure 3 (b) using the 
(a)

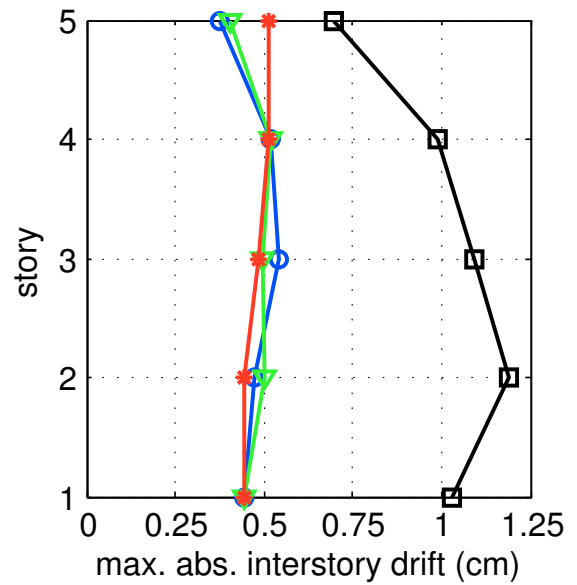

(b)

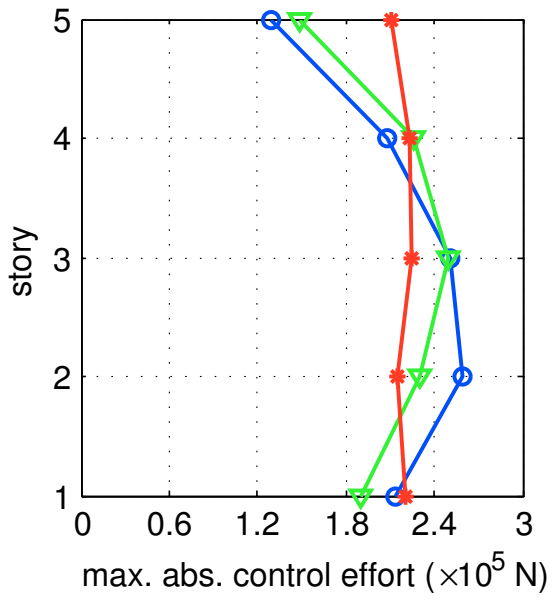

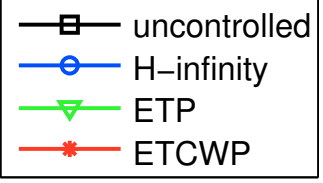

Figure 3: Response of the five-story building model corresponding to the scaled North-South El Centro 1940 seismic record for the uncontrolled configuration (black line with squares), the $H_{\infty}$ controller (blue line with circles), the energy-to-peak controller (green line with triangles) and the energy-to-componentwise-peak controller (red line with asterisks). (a) Maximum absolute interstory drifts. (b) Maximum absolute control efforts.

\begin{tabular}{lcccccc}
\hline story & $1-5$ & $6-11$ & $12-14$ & $15-17$ & $18-19$ & 20 \\
\hline mass $\left(\times 10^{6} \mathrm{Kg}\right)$ & 1.10 & 1.10 & 1.10 & 1.10 & 1.10 & 1.10 \\
stiffness $\left(\times 10^{8} \mathrm{~N} / \mathrm{m}\right)$ & 8.62 & 5.54 & 4.54 & 2.91 & 2.56 & 1.72 \\
relative damping & $5 \%$ & & & & & \\
\hline
\end{tabular}

Table 3: Parameter values corresponding to the twenty-story building model.

same colors and symbols.

Looking at the matrices in (45), 46) and 47) it can be appreciated that the proposed design methods certainly produce different results. A quick inspection of the plots in Figure 3 reveals two clear facts: (i) the three controllers provide a good level of seismic protection with similar levels of control effort and (ii) the energy-to-componentwise-peak controller exhibits a particularly well-balanced behavior. The computation times in Table 2 also indicate two facts: (i) all the proposed design strategies demand very small computation times and, consequently, are suitable for control problems involving small-size building models and (ii) the shortest computation time is attained by the energy-to-peak controller.

Now we consider the twenty-story building model [10] corresponding to the mass, stiffness and damping parameters presented in Table 3 . For this large-building problem, we compute an $H_{\infty}$ controller by solving the LMI optimization problem $\mathcal{P}_{\infty}$ in (13) with the matrices $A, B$ and $E$ corresponding to the values in Table 3 and the controlled-output matrices in (44) with $n=20$ and the scaling factor $\alpha=10^{-8.0}$. We also design an energy-to-peak controller and an energyto-componentwise-peak controller by solving the optimization problems $\mathcal{P}_{\mathrm{p}}$ in $(18)$ and $\mathcal{P}_{\text {cwp }}$ in (25), respectively, with the same matrices $A, B, E$ and $C$ used in the $H_{\infty}$ design and the matrix $D$ defined by $n=20$ and the scaling factor $\alpha=10^{-8.2}$. The computation times corresponding to this second set of controllers are collected in Table 4. To illustrate the seismic performance of the proposed design strategies in this harder control problem, the twenty-story building vibration response has been numerically simulated using also the scaled North-South El Centro 1940 
(a)

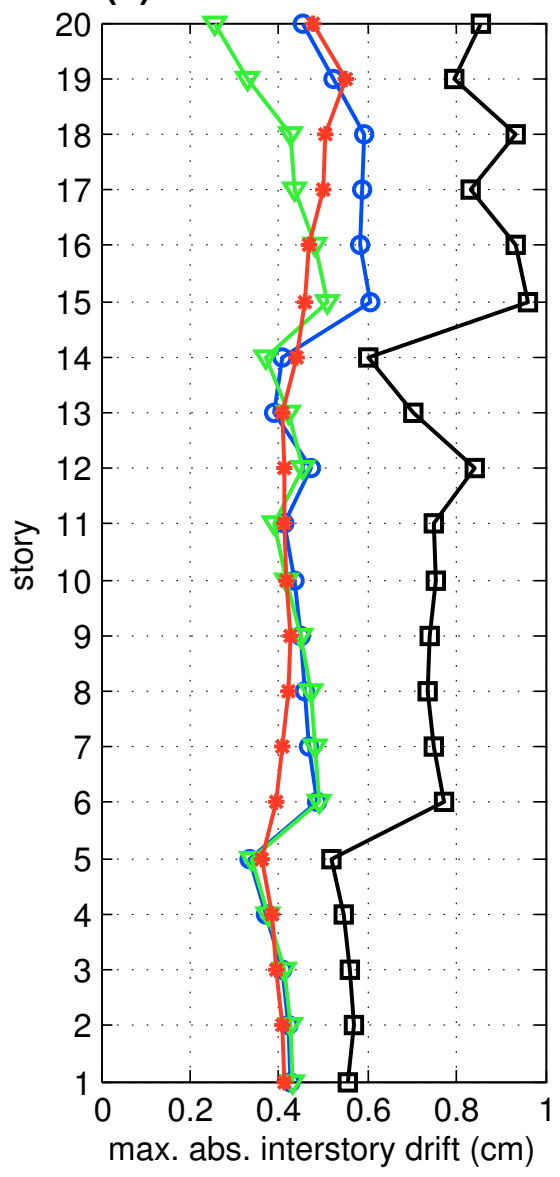

(b)

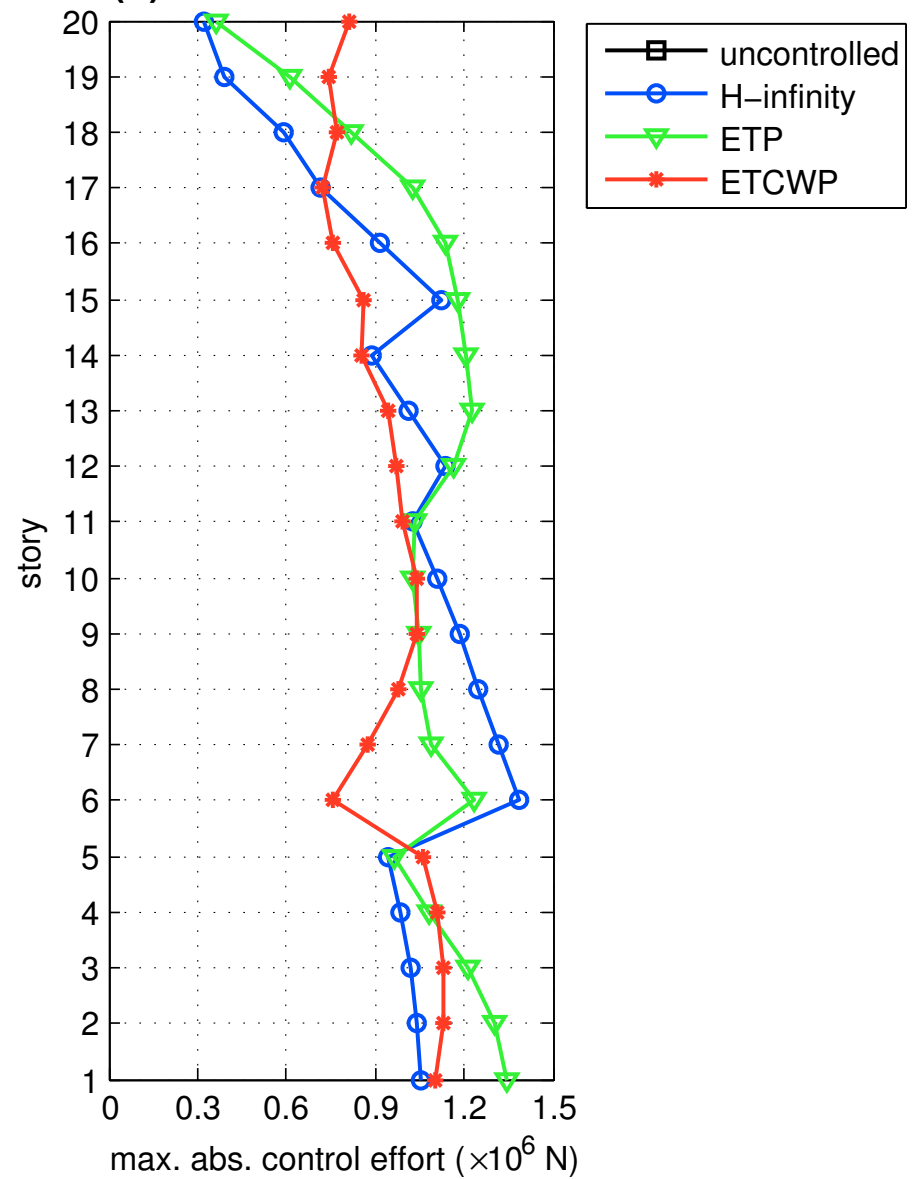

Figure 4: Response of the twenty-story building model corresponding to the scaled North-South El Centro 1940 seismic record for the uncontrolled configuration (black line with squares), the $H_{\infty}$ controller (blue line with circles), the energy-to-peak controller (green line with triangles) and the energy-to-componentwise-peak controller (red line with asterisks). (a) Maximum absolute interstory drifts. (b) Maximum absolute control efforts.

\begin{tabular}{lccc}
\hline controller & $H_{\infty}$ & ETP & ETCWP \\
\hline computation time (s) & 169.25 & 60.63 & 458.24 \\
\hline
\end{tabular}

Table 4: Computation time (in seconds) corresponding to the $H_{\infty}$, energy-to-peak (ETP) and energy-tocomponentwise-peak (ETCWP) controllers designed for the twenty-story building model.

seismic record as ground acceleration input. The corresponding absolute interstory-drift and absolute control-effort peak-values are displayed in Figure 4(a) and Figure4(b), respectively, using the same colors and symbols: black line with squares for the uncontrolled response, blue line with circles for the $H_{\infty}$ controller, green line with triangles for the energy-to-peak controller and red line with asterisks for the energy-to-componentwise-peak controller.

The inspection of the plots in Figure 4 indicates that the same two facts observed in the five-story building problem still hold in the present case: (i) the three controllers provide a good level of seismic protection with similar levels of control effort and (ii) a particularly wellbalanced behavior is exhibited by the energy-to-componentwise-peak controller. Looking at the 
computation times in Table 4 and comparing with the data in Table 2 , three main facts can be appreciated: (i) the best results are still attained by the energy-to-peak controller, (ii) all computation times are significantly larger and, (iii) a particularly larger computation time is required by the energy-to-componentwise-peak controller. The good computational performance of the energy-to-peak design strategy is a remarkable fact that converts this design methodology in an interesting option for vibration control of large structures. The observed increment of the computation times can be partially explained by the increase in the number of LMI variables. It should be observed that, in the general case of a $n$-story building with $n$ actuation devices, the dimensions of the LMI variable matrices $X$ and $Y$ are $2 n \times 2 n$ and $n \times 2 n$, respectively, and the total number of variables in the considered LMI optimization problems is $4 n^{2}+n$. This means that a controller design for a five-story building involves 105 LMI variables while a twentystory building design requires 1620 LMI variables. Additionally, the larger computation time required by the energy-to-componentwise-peak design in the twenty-story building problem is consistent with the higher complexity of the corresponding LMI formulation.

\section{CONCLUSIONS}

In this paper, we have conducted a comparative study of three LMI-based controller design strategies for structural vibration control, paying special attention to the computational effectiveness in large-dimension problems. Together with the well-known $H_{\infty}$ approach, the study includes the energy-to-peak and energy-to-componentwise-peak methodologies, which are particular cases of generalized $\mathrm{H}_{2}$ designs. These three controller design strategies have been applied to synthesize active state-feedback controllers for the seismic protection of a five-story building and a twenty-story building, both equipped with a complete system of interstory actuation devices. The corresponding computation times have been considered to evaluate the computational effectiveness of the proposed design strategies and a proper set of numerical simulations has been carried out to assess the performance of the obtained controllers. In the five-story building problem, all the proposed design strategies have demanded computation times inferior to one second and the obtained controllers provide a good level of seismic protection with similar levels of control effort. In the twenty-story building problem, a good level of performance is also attained by all the controllers but an important increment of the computation times has been observed and significant differences can be appreciated in the computational effectiveness of the different design strategies. Overall, the following three main facts can be highlighted: (i) significantly shorter computation times are required by the synthesis of energy-to-peak controllers, (ii) a particularly well-balanced behavior is exhibited by the energy-to-componentwise-peak controllers and (iii) important computational difficulties should be expected when applying the considered controller design strategies to very-large-scale control problems.

\section{ACKNOWLEDGMENTS}

This work was partially supported by the Spanish Ministry of Economy and Competitiveness under Grant DPI2015-64170-R.

\section{REFERENCES}

[1] S. Boyd, L. El Ghaoui, E. Feron, and V. Balakrishnan. Linear Matrix Inequalities in System and Control Theory. SIAM, Philadelphia, USA, 1994. 
[2] G.W. Housner, L.A. Bergman, T.K. Caughey et al. Structural control: Past, present, and future. Journal of Engineering Mechanics, 123(9):897-971, 1997.

[3] B.F. Spencer and S. Nagarajaiah. State of the art of structural control. Journal of Structural Engineering, 129(7):845-856, July 2003.

[4] S.Y. Chu, T.T. Soong, and A.M. Reinhorn. Active, Hybrid and Semi-Active Structural Control. Wiley, 2005.

[5] Y. Ikeda. Active and semi-active vibration control of buildings in Japan - Practical applications and verification. Structural Control and Health Monotoring, 16(7-8):703-723, 2009.

[6] H. Li and L. Huo. Advances in structural control in civil engineering in China. Mathematical Problems in Engineering, pages 1-23, 2010.

[7] A. Zečević and D.D. Šiljak. Control of Complex Systems. Structural Constraints and Uncertainty. Springer, 2010.

[8] G.J. Balas, R.Y. Chiang, A.K. Packard, and M.G. Safonov. MATLAB ${ }^{T M}$ Robust Control Toolbox 3. User's Guide, Version 4.2. The MathWorks, Inc., 3 Apple Hill Drive. Natick, MA 01760-20, USA, 2012.

[9] H. Du, J. Lam, and K.Y. Sze. Non-fragile $H_{\infty}$ vibration control for uncertain structural systems. Journal of Sound and Vibration, 273(4-5):1031-1045, 2004.

[10] Y. Wang, J.P. Lynch, and K.H. Law. Decentralized $H_{\infty}$ controller design for large-scale civil structures. Earthquake Engineering and Structural Dynamics, 38(3):377-401, 2009.

[11] J.M. Rossell, F. Palacios-Quiñonero, and J. Rodellar. Semi-decentralized output feedback $H_{\infty}$ control strategy for large building structures. In Proceedings of the 5th World Conference on Structural Control and Monitoring (5WCSCM), Shinjuku, Japan, July 2010.

[12] Y. Wang. Time-delayed dynamic output feedback $H_{\infty}$ controller design for civil structures: A decentralized approach through homotopic transformation. Structural Control and Health Monotoring, 18(2):121-139, 2011.

[13] F. Palacios-Quiñonero, J. Rubió-Massegú, J.M. Rossell, and H.R. Karimi. Discrete-time static output-feedback semi-decentralized $H_{\infty}$ controller design: an application to structural vibration control. In Proceedings of the 2012 American Control Conference, pages 6126-6131, Montréal, Canada, 2012.

[14] J. Rubió-Massegú, F. Palacios-Quiñonero, and J.M. Rossell. Decentralized static outputfeedback $H_{\infty}$ controller design for buildings under seismic excitation. Earthquake Engineering and Structural Dynamics, 41(7):1199-1205, 2012.

[15] F. Palacios-Quiñonero, J. Rubió-Massegú, J.M. Rossell, and H.R. Karimi. Feasibility issues in static output-feedback controller design with application to structural vibration control. Journal of the Franklin Institute, 351(1):139-155, 2014.

[16] M.A. Rotea. The generalized $H_{2}$ control problem. Automatica, 29(2):373-385, 1993. 
[17] H. Du and J. Lam. Energy-to-peak performance controller design for building via static output feedback under consideration of actuator saturation. Computers and Structures, 84(31-32):2277-2290, 2006.

[18] W. Zhang, Y. Chen, and H. Gao. Energy-to-peak control for seismic-excited buildings with actuator faults and parameter uncertainties. Journal of Sound and Vibration, 330(4):581$602,2011$.

[19] F. Palacios-Quiñonero, J. Rubió-Massegú, J.M. Rossell, and H.R. Karimi. Vibration control for adjacent structures using local state information. Mechatronics, 24(4):336-344, 2014.

[20] F. Palacios-Quiñonero, J. Rubió-Massegú, J.M. Rossell, and H.R. Karimi. Optimal design of complex passive-damping systems for vibration control of large structures: an energyto-peak approach. Abstract and Applied Analysis, pages 1-9, 2014.

[21] J.N. Yang, S. Lin, and F. Jabbari. Linear multi-objective control strategies for wind-excited buildings. Journal of Engineering Mechanics, 130(4):471-477, 2004.

[22] A.K. Chopra. Dynamics of Structures. Theory and Applications to Earthquake Engineering. Prentice Hall, Upper Saddle River, New Jersey, USA, 3rd edition, 2007.

[23] N. Kurata, T. Kobori, M. Takahashi, N. Niwa, and H. Midorikawa. Actual seismic response controlled building with semi-active damper system. Earthquake Engineering and Structural Dynamics, 28(11):1427-1447, 1999. 